\title{
Validez de la escala de Braden para predecir úlceras por presión en población femenina
}

\author{
Juan E Blümel M, Karina Tirado Ga, Claudia Schiele $M^{a}$, \\ G abriela Schönffeldt Fa, Salvador Sarrá C. \\ Prediction of the pressure ulcer \\ development in elderly women using \\ the Braden Scale
}

Background: Pressure ulcers are a common complication among elderly patients confined to bed for long periods. The Braden scale is a commonly used risk assessment tool. Aim: To evaluate the use of Braden scale. Patients and methods: Seventy women aged 61 to 96 years, admitted to the Internal Medicine Service of Barros Luco-Trudeau Hospital, were studied. Their risk was evaluated using the Braden scale. The presence of pressure ulcer was diagnosed according to the National Pressure Ulcer Advisory Panel on admition, two weeks later and at discharge. Results: On admission, mean Braden scale score was $16.6 \pm 2.8$ and 34 women had a score of 16 or less, that is considered of risk. Twenty five women (20 with a score of 16 or less) developed pressure ulcers, mostly superficial. The odds ratio of a score of 16 or less for the development of ulcers was 4.2 (95\% CI 1.8-11.7, p <0.001). The sensitivity and specificity of such score were 80 and $69 \%$ respectively. Conclusions: The Braden scale predicts the risk of developing pressure ulcers with a good sensitivity and specificity in female elderly patients (Rev Méd Chile 2004; 132: 595-600).

(Key Words: Aged; Decubitus ulcer; Skin ulcer)

Recibido el 13 de junio, 2003. Aceptado en versión corregida el 15 de marzo, 2004. Departamento Medicina Sur, Universidad de Chile. Santiago de Chile.

aAlumnas V año de Medicina, Universidad de Chile

L as úlceras por presión son producidas por la _prolongada presión ejercida por un objeto externo sobre prominencias óseas, provocando ulceración y necrosis del tejido involucrado. Se presentan con mayor frecuencia en la región sacra, los talones, las tuberosidades isquiáticas y las caderas. Entre los principales factores de riesgo que favorecen su desarrollo se encuentran: edad, déficit nutricional, inmovilidad, fricción, diabetes,

Correspondencia a: Karina Tirado. Fono: 09-7191839.

E-mail: tivurrtyra@hotmail.com estado mental y humedad ${ }^{1-6}$. En general, los pacientes que más frecuentemente presentan úlceras por presión son aquellos de edad avanzada, postrados por períodos prolongados en condiciones de inmovilidad. Esta situación se observa principalmente en los servicios de cuidados intensivos o neurología. La tendencia a infectarse que tienen estas lesiones provoca, no solamente problemas de salud al paciente, sino que también una prolongación de la estadía hospitalaria y un incremento en los costos de hospitalización? Además, la incidencia de las úlceras por presión, 
como reconoce la OMS, refleja la calidad asistencial de la red hospitalaria de un país 8 .

Para una identificación objetiva y precisa del riesgo de presentar úlceras por presión se han desarrollado diversas escalas ${ }^{9}$. Una de las más utilizadas es la de Braden, la cual ha sido validada en diferentes escenarios ${ }^{10-12}$. Para predecir la población en riesgo de desarrollar úlceras por presión $\mathrm{y}$, de esta manera, manejar en forma más focalizada los recursos médicos y de enfermería, diseñamos un estudio con el objeto de evaluar en nuestro medio la utilidad de instrumento de Braden.

\section{MATERIALES Y MÉTODOS}

Pacientes. Criterios de inclusión: Todas las mujeres mayores de 60 años que ingresen al Servicio de Medicina del Hospital Barros Luco-Trudeau, entre agosto y octubre de 2002. Criterios de exclusión: Pacientes que a su ingreso al Hospital presenten úlceras por presión.

El Hospital Barros Luco-Trudeau es un centro de referencia que recibe a la población adulta del sector sur de Santiago, estimada en 1.200.000 habitantes.

Diseño del estudio. El diseño de esta investigación corresponde a un estudio prospectivo observacional en que se evaluó al ingreso al Hospital el riesgo de desarrollar úlceras por presión con la Escala de Braden. Se buscó la presencia de úlceras por presión al ingreso, a las dos semanas y al egreso.

\section{Instrumentos}

Escala de Braden (Figura 1). Evalúa el riesgo de desarrollar úlceras por presión en base a la sensibilidad y humedad de la piel, la fricción del cuerpo con las sábanas, la movilidad, actividad y nutrición del paciente. Un puntaje menor o igual a

Figura 1. Escala de Braden

\begin{tabular}{|c|c|c|c|c|}
\hline $\begin{array}{l}\text { Percepción Sensorial } \\
\text { Capacidad de respuesta } \\
\text { a estímulos dolorosos }\end{array}$ & $\begin{array}{l}\text { 1. Limitado } \\
\text { completamente }\end{array}$ & $\begin{array}{l}\text { 2. Muy } \\
\text { limitado }\end{array}$ & $\begin{array}{l}\text { 3. Limitado } \\
\text { levemente }\end{array}$ & $\begin{array}{l}\text { 4. Sin } \\
\text { impedimento }\end{array}$ \\
\hline \multicolumn{5}{|l|}{ Humedad } \\
\hline Grado de humedad de piel & $\begin{array}{l}\text { 1. Constantemente } \\
\text { húmeda }\end{array}$ & $\begin{array}{l}\text { 2. Muy } \\
\text { húmeda }\end{array}$ & $\begin{array}{l}\text { 3. Ocasionalmente } \\
\text { húmeda }\end{array}$ & $\begin{array}{l}\text { 4. Raramente } \\
\text { húmeda }\end{array}$ \\
\hline \multicolumn{5}{|l|}{ Actividad } \\
\hline Grado de actividad física & $\begin{array}{l}\text { 1. Confinado } \\
\text { a la cama }\end{array}$ & $\begin{array}{l}\text { 2. Confinado } \\
\text { a la silla }\end{array}$ & $\begin{array}{l}\text { 3. Ocasionalmente } \\
\text { camina }\end{array}$ & $\begin{array}{l}\text { 4. Camina } \\
\text { frecuentemente }\end{array}$ \\
\hline \multicolumn{5}{|l|}{ Movilidad } \\
\hline Control de posición corporal & $\begin{array}{l}\text { 1. Completamente } \\
\text { inmóvil }\end{array}$ & $\begin{array}{l}\text { 2. Muy } \\
\text { limitada }\end{array}$ & $\begin{array}{l}\text { 3. Levemente } \\
\text { limitada }\end{array}$ & $\begin{array}{l}\text { 4. Sin } \\
\text { limitaciones }\end{array}$ \\
\hline \multicolumn{5}{|l|}{ Nutrición } \\
\hline Patrón de ingesta alimentaria & $\begin{array}{l}\text { 1. Completamente } \\
\text { inadecuada }\end{array}$ & $\begin{array}{l}\text { 2. Probablemente } \\
\text { inadecuada }\end{array}$ & 3. Adecuada & 4. Excelente \\
\hline \multicolumn{5}{|l|}{ Fricción y roce } \\
\hline Roce de piel con sábanas & 1. Presente & $\begin{array}{l}\text { 2. Potencialmente } \\
\text { presente }\end{array}$ & 3. Ausente & \\
\hline
\end{tabular}

Se considera como riesgo de desarrollar úlceras un puntaje menor o igual a 16. 
Figura 2. Clasificación de las escaras.

National Pressure U Icer Advisory Panel

\begin{tabular}{|ll|}
\hline Etapa & Descripción \\
\hline I & Eritema no blanqueable en piel intacta \\
II & Pérdida parcial del grosor de la piel, involucrando epidermis y dermis \\
III & $\begin{array}{l}\text { Pérdida total del grosor de la piel, involucrando daño o necrosis del tejido subcutáneo sin } \\
\text { abarcar estructuras subyacentes o fascia }\end{array}$ \\
IV & $\begin{array}{l}\text { Pérdida total del grosor de la piel con daño de estructuras subyacentes de soporte tales } \\
\text { como fascia, tendones, cápsula articular, etc. }\end{array}$ \\
\hline
\end{tabular}

16 implica riesgo de desarrollar úlceras por presión, se utilizó este punto de corte ya que es lo que se describe por los autores de la escala para su utilización ${ }^{13}$.

National Pressure Ulcer Advisory Panel. Instrumento mediante el cual las úlceras por presión son etapificadas en cuatro grados según su profundidad (Figura 2). Mayor información sobre esta clasificación puede encontrarse en las referencias 14 y 15.

Estadística. Los datos fueron analizados con el programa Epi-Info 6.04. Los resultados fueron analizados según el riesgo relativo. Se considera significación estadística a $\mathrm{p}<0,05$. Las diferencias entre los promedios de riesgo de los diferentes grupos fueron analizados mediante $\mathrm{T}$ de Student. Los resultados se expresan como media de desviación estándar.

\section{RESULTADOS}

La población estudiada en este trabajo corresponde a 70 pacientes ingresados al Servicio de Medicina del Hospital Barros Luco-Trudeau mayo-
Tabla 1. Edad de las pacientes estudiadas

\begin{tabular}{|lcc|}
\hline Edad & $\begin{array}{c}\mathrm{N}^{\circ} \text { de } \\
\text { pacientes }\end{array}$ & $\begin{array}{c}\text { Porcentaje } \\
(\%)\end{array}$ \\
\hline $60-64$ & 10 & 14,3 \\
$65-69$ & 13 & 18,6 \\
$70-74$ & 12 & 17,1 \\
$75-79$ & 16 & 22,9 \\
$80-84$ & 9 & 12,9 \\
$\geq 85$ & 10 & 14,2 \\
Total & 70 & 100 \\
\hline
\end{tabular}

res de 60 años, todas de sexo femenino. La edad promedio de las pacientes fue de $74,7 \pm 8,7$ años, con un rango de 61-96 años (Tabla 1).

Utilizando la escala de Braden, se obtiene que el promedio de puntaje de estas mujeres a su ingreso era de 16,6 $\pm 2,8$. Al aplicar el punto de corte que los autores del test consideran de riesgo, menor o igual a 16 , obtenemos que $48,6 \%$ de las mujeres pueden definirse como en riesgo de desarmollar úlceras por presión (Tabla 2). Del total de pacientes estudiadas,

Tabla 2. Pacientes que desarrollaron escaras según puntaje de Braden

\begin{tabular}{|c|c|c|c|c|c|c|}
\hline \multirow{2}{*}{ Puntaje Braden (Riesgo) } & \multicolumn{2}{|c|}{ Con escaras } & \multicolumn{2}{|c|}{ Sin escaras } & \multicolumn{2}{|c|}{ Total } \\
\hline & $\mathrm{n}$ & $\%$ & $\mathrm{n}$ & $\%$ & $n$ & $\%$ \\
\hline$>16$ puntos (sin riesgo) & 5 & 13,9 & 31 & 86,1 & 36 & 51,4 \\
\hline$\leq 16$ puntos (con riesgo) & 20 & 58,8 & 14 & 41,2 & 34 & 48,6 \\
\hline Total & 25 & 35,7 & 45 & 64,3 & 70 & 100,0 \\
\hline
\end{tabular}

RR: 4,235. IC: 1,783-11,743. $\mathrm{p}<0,0001$. 
veinticinco $(35,7 \%)$ presentaron úlceras por presión. Más de la mitad (58,8\%) de las mujeres con riesgo, según la escala de Braden, desarmollaron úlceras por presión durante el período de observación. Un puntaje inferior o igual a 16 implicó una probabilidad cuatro veces mayor de tener úlceras por presión (RR: 4,235 IC: 1,783-11,743 p «0,0001). La sensibilidad del test en nuestras pacientes fue de $80 \%$, la especificidad de $68,9 \%$, el valor predictivo positivo de $58,8 \%$ y el valor predictivo negativo fue de $86,1 \%$.

La mayor parte de las pacientes (80\%) presentaron úlceras por presión superficiales (Tabla 3). Al evaluar la etapificación de las úlceras por presión y relacionarla con el riesgo, se observó que las pacientes que presentaron úlceras por presión en etapa I tenían un puntaje promedio de riesgo de 15,1 $\pm 1,7$; en aquellas con úlceras por presión en etapa II el promedio fue de 12,5 $\pm 2,1$ y las con úlceras por presión en etapa III tenían un promedio de $11,0 \pm 1,0$ ( $p<0,001$ ). El puntaje promedio de riesgo que presentaron el total de pacientes que desarrollaron úlceras por presión fue de $14,4 \pm 2,17$, mientras que aquellas pacientes que no desarrollaron úlceras por presión tuvieron un puntaje promedio de riesgo de $17,77 \pm 2,295$; la diferencia de estos promedios es significativa según la prueba de t-Student (tcalc: 6,02 á: 0,05).

El promedio de días cama fue de 10,5 $\pm 9,174$ con un rango entre 3-67 días.

\section{DisCUSIÓN}

En Chile, se han realizado pocos trabajos respecto a la incidencia y prevención del desarrollo de úlceras por presión, a pesar de la importancia médica y económica que estas lesiones implican ${ }^{7,16}$. Nuestros resultados muestran que la incidencia de úlceras por presión es un problema frecuente en las mujeres hospitalizadas de edad avanzada, segmento etario reconocido de mayor riesgo de presentar úlceras por presión $2,3,5$ y que presentan, además, una alta tasa de hospitalización.

El contar con un instrumento predictivo de riesgo de desarrollar úlceras por presión, se considera de gran utilidad para poder realizar una mejor prevención ${ }^{17}$. Los resultados obtenidos por nosotros demuestran que, en nuestro medio, la escala de Braden es un instrumento capaz de predecir el
Tabla 3. Puntajes en la escala de Braden según profundidad de las escaras (N PUAP*)

\begin{tabular}{|lrrc|}
\hline $\begin{array}{l}\text { Profundidad } \\
\text { escaras (etapas) }\end{array}$ & \multicolumn{2}{c}{ Pacientes } & $\begin{array}{c}\text { Puntaje } \\
\text { de riesgo }\end{array}$ \\
\hline Sin escaras & 45 & 64,28 & $17,77 \pm 2,295$ \\
\hline I & 20 & 28,57 & $15,1 \pm 1,7$ \\
II & 2 & 2,85 & $12,5 \pm 2,1$ \\
III & 3 & 4,28 & $11,0 \pm 1,0$ \\
\hline
\end{tabular}

* National Pressure Ulcer Advisory Panel.

riesgo de desarrollar úlceras por presión con una adecuada sensibilidad y especificidad. Sin embargo, los resultados de otros trabajos muestran un amplio rango de sensibilidades (entre 35-100\%) debido, probablemente, a que fueron realizados en distintos ambientes y con poblaciones diferentes, por ejemplo, en pacientes hospitalizados, ancianos en casas de reposo, en atención primaria, etc ${ }^{9-12}$. El puntaje de corte para clasificar a los pacientes en grupos con y sin riesgo de desarrollar úlceras por presión no ha sido bien definido, diferentes estudios utilizan distintos puntos de corte ${ }^{11,18}$, el punto de corte utilizado en nuestro estudio fue de 16, que es el recomendado por los autores del instrumento y que además se encuentra avalado en diversos estudios, presentando una sensibilidad de $100 \%{ }^{12,19}$. Otro factor que influye, es la definición y la etapificación de las úlceras por presión que usan los diferentes estudios, habiendo algunos que encuentran como significativas las úlceras por presión sólo de etapa 2 en adelante, quizás por la mayor dificultad de reconocer una úlceras por presión en etapa 1, lo cual creemos no es adecuado, ya que las úlceras por presión etapa 1 son las que se presentan con mayor frecuencia 20,$21 ; y$, si bien son reversibles en la mayonía de los casos, son un importante signo de alarma para el personal de salud y el paciente, que permitiría actuar de manera preventiva, evitando su progresión a grados mayores, y por ende, sus complicaciones. Esto es muy importante de considerar, ya que lo que realmente determinará el desarrollo de una úlcera por presión, serán los cuidados a los que se someta un paciente en riesgo. Si se interviniese en todos 
los factores que facilitan el desarrollo de estas lesiones, los pacientes no desarrollarían finalmente úlceras por presión, lo que podría explicar por qué un grupo de pacientes que tenía riesgo elevado en este estudio no las desarrolló. Podemos deducir entonces, que la presencia de úlceras por presión está muy relacionado con los cuidados que se les dé a los pacientes, y la escala de Braden nos permite clasificar a qué pacientes se les debe proporcionar más cuidados.

Un resultado interesante en nuestro trabajo fue el hecho de que el puntaje obtenido en la Escala de Braden se relaciona directamente con la gravedad de las úlceras por presión. Este hecho nos muestra que, con medidas de prevención, en aquellos pacientes con mayor riesgo según esa escala, no sólo se podría impedir el desarrollo de úlceras por presión, sino que además podríamos evitar la aparición de lesiones profundas, las que son de más difícil manejo y tienen mayor tendencia a complicarse.

En nuestro estudio, la gran proporción de úlceras por presión en etapa 1 se debería al medio donde realizamos el trabajo, el Servicio de Medicina, ya que se trataría de pacientes de menor gravedad y con menos factores de riesgo en comparación con servicios de mayor complejidad. Esto se ve reflejado en que las pacientes no presentaban puntajes extremadamente bajos, a

\section{REFERENCIAS}

1. Schimdt T. Pressure ulcers. Nutrition strategies that make a difference. Caring 2002; 21: 18-24.

2. Perneger TV, Rae AC, Gaspoz JM, Borst F, Vitek O, Нецот C. Screening for pressure ulcer risk in an acute care hospital: development of a brief bedside scale. J Clin Epidemiol 2002; 55: 498-504.

3. Casimiro C, García de Lorenzo A, Usan L Prevalence of decubitus ulcer and associated risk factors in an institutionalized Spanish elderly population. Nutrition 2002; 18: 408-14.

4. SoNG M, CHOI KS. Factors predicting development of decubitus ulcers among patients admitted for neurological problems. Kanho Hakhoe Chi 1991; 21: $16-26$.

5. Bours GJ, de laat E, Halfens RJ, Lubbers M. Prevalence, risk factors and prevention of pres- pesar de presentar un cierto nivel de riesgo. Es por esta misma razón que creemos que registramos sólo tres pacientes con úlceras por presión en etapa III y ninguna, en etapa IV.

En muchos estudios describen que el sitio más frecuente de úlceras por presión es la sacra ${ }^{22-24}$. Nuestros resultados muestran que la localización más observada en estas pacientes fue en los talones. Esta diferencia es difícil de interpretar, pero podńa deberse a la definición que se use de úlceras por presión. Las sacras son en general más profundas. En nuestro estudio predominaron las úlceras por presión en los talones que son más superficiales.

Podríamos concluir señalando que la escala de Braden es un instrumento capaz de predecir el riesgo de desarrollar úlceras por presión en la población femenina con una adecuada sensibilidad y especificidad. Es, además, un instrumento de fácil aplicación, por usar conceptos claros y bien definidos, que al aplicarse en el momento de ingreso de los pacientes podría servir como una guía para disponer eficazmente de los recursos humanos y técnicos en aquellos pacientes en riesgo de desarrollar úlceras por presión. Su utilidad en la prevención del desarrollo y complicación de úlceras por presión traerá un menor costo económico y mayor beneficio en la atención de los pacientes. Por esto, su aplicación clínica debiera ser recomendada.

sure ulcers in Dutch intensive care units. Results of a cross-sectional survey. Intensive Care Med 2001; 27: 1599-605.

6. Auman RM. Pressure ulcer prevalence, incidence, risk factors and impact. Clin Geriatr Med 1997; 13: 421-36.

7. Aluman RM, Goode PS, Burst N, Bartolucci AA, ThomAs DR. Pressure ulcers, hospital complications, and disease severity: impact on hospital cost and lenght of stay. Advances in Wound Care 1999; 12: 22-30.

8. Estudio sobre úlceras por presión en un centro socio-sanitario, Alfons Cordomí Gotanegra. http:/ / www.dragonet.es/users/d1346/nafres.htm, 06 de enero de 2004.

9. Boes C. Reliability and validity of the Braden Scale for predicting pressure sore risk. Pflege 2000; 13: 397-402. 
10. Pang S, Wong T. Predicting pressure sore risk with the Norton, Braden and Waterlow scales in a Hong Kong rehabilitation hospital. Nursing Research 1998; 47: 147-53.

11. RAMUNDO JM. Reliability and validity of the Braden Scale in the home care setting. J Wound Ostomy Continence Nurs 1995; 22: 128-34.

12. Barnes D, PaYton RG. Clinical application of the Braden Scale in the acute care. Dermatol Nurs 1993; 5: 386-8.

13. Braden Risk Assessment Scale. http:// www.webmedtechnology.com/public/BradenScale-skin.pdf, 06 de enero de 2004.

14. Ulcer classification. http://woundcare.org/ newsvol2n2/ulcer1.htm\#NPUAP, 06 de enero de 2004.

15. Pressure care for pressure ulcer and pain therapy. http://www.pressure-care.co.uk/guides/bedsores-wounds-pictures-of-decubitus-ulcerstages.htm, 06 de enero de 2004.

16. LIVESLEY NJ, CHOw AW. Infected pressure ulcers in elderly individuals. Clin Infect Dis 2002; 35: 13906.

17. Mazzocco R, Zampieron A. Does the evaluation of the pressure ulcer risk increase better prevention? Prof Inferm 2000; 53: 173-8.
18. Bergstrom N, Braden B, Kemp M, Champagne M, RuBY E. Predicting pressure ulcer risk: a multisite study of the predictive validity of the Braden Scale. Nurs Res 1998; 47: 261-9.

19. Bergstrom N, Braden BJ, Laguzza A, Holman V. The Braden Scale for predicting pressure sore risk. Nurs Res 1987; 36: 205-10.

20. Thoroddsen A. Pressure sore prevalence: a national survey. J Clin Nurs 1999; 8: 170-9.

21. Halfens RJ, Bour GJ, VAn Ast W. Relevance of the diagnosis "stage 1 pressure ulcer": an empirical study of the clinical course of stage 1 ulcers in acute care and long term-care hospital populations. Journal of Clinical Nursing 2001; 10: 748-57.

22. De Astis V, Corbe山a A, Bafico F, Sinem E, Porcu G, Bottari L et al. Decubitus lesions in patients referred to acute and post-acute home nursing care for the eldery in Génova. Assistenza infermieristica e ricerca 1999; 18: 20-4.

23. Bours GJ, Halfens RJ, Lubbers M, Haalboom JR. The development of a national registration form to measure the prevalence of pressure ulcers in The Netherlands. Ostomy Wound Manage 1999; 45: 28-33, 36-8, 40.

24. Yarkony GM. Pressure ulcers: a review. Arch Phys Med Rehabil 1994; 75: 908-17. 